\title{
Effects of Green Tea Catechins and Theanine on Preventing Influenza Infection among Healthcare Workers: A Randomized Controlled Trial
}

Keiji Matsumoto ${ }^{1 \dagger}$, Hiroshi Yamada ${ }^{1{ }^{* \dagger}}$, Norikata Takuma ${ }^{2 \dagger}$, Hitoshi Niino ${ }^{3 \dagger}$, Yuko M Sagesaka ${ }^{3+}$

\begin{abstract}
Background: Experimental studies have revealed that green tea catechins and theanine prevent influenza infection, while the clinical evidence has been inconclusive. This study was conducted to determine whether taking green tea catechins and theanine can clinically prevent influenza infection.

Methods: Design, Setting, and Participants: A randomized, double-blind, placebo-controlled trial of 200 healthcare workers conducted for 5 months from November 9, 2009 to April 8, 2010 in three healthcare facilities for the elderly in Higashimurayama, Japan.

Interventions: The catechin/theanine group received capsules including green tea catechins (378 mg/day) and theanine $(210 \mathrm{mg} /$ day). The control group received placebo.

Main Outcome Measures: The primary outcome was the incidence of clinically defined influenza infection. Secondary outcomes were (1) laboratory-confirmed influenza with viral antigen measured by immunochromatographic assay and (2) the time for which the patient was free from clinically defined influenza infection, i.e., the period between the start of intervention and the first diagnosis of influenza infection, based on clinically defined influenza infection.

Results: Eligible healthcare workers $(n=197)$ were enrolled and randomly assigned to an intervention; 98 were allocated to receive catechin/theanine capsules and 99 to placebo. The incidence of clinically defined influenza infection was significantly lower in the catechin/theanine group (4 participants; $4.1 \%$ ) compared with the placebo group (13 participants; 13.1\%) (adjusted $\mathrm{OR}, 0.25 ; 95 \% \mathrm{Cl}, 0.07$ to $0.76, P=0.022$ ). The incidence of laboratory-confirmed influenza infection was also lower in the catechin/theanine group (1 participant; 1.0\%) than in the placebo group (5 participants; 5.1\%), but this difference was not significant (adjusted OR, 0.17; $95 \% \mathrm{Cl}, 0.01$ to $1.10 ; P=0.112$ ). The time for which the patient was free from clinically defined influenza infection was significantly different between the two groups (adjusted HR, 0.27; 95\% Cl, 0.09 to 0.84; $P=0.023)$.
\end{abstract}

Conclusions: Among healthcare workers for the elderly, taking green tea catechins and theanine may be effective prophylaxis for influenza infection.

Trial Registration: ClinicalTrials (NCT): NCT01008020

\footnotetext{
* Correspondence: hyamada@u-shizuoka-ken.ac.jp

† Contributed equally

${ }^{1}$ Department of Drug Evaluation \& Informatics, Graduate School of

Pharmaceutical Sciences, University of Shizuoka, 52-1 Yada, Suruga-ku,

Shizuoka 422-8526, Japan

Full list of author information is available at the end of the article
} 


\section{Background}

Influenza infection is the principal cause of acute respiratory illnesses and occurs in epidemics worldwide, in all ages [1]. To reduce morbidity and mortality, a variety of public health interventions have been implemented, including facemasks, gargling, improved hand hygiene and coughing etiquette [2-5]. Vaccines are the most widely used intervention for influenza infection prophylaxis, but their effectiveness depends on the type of influenza virus involved in each season's epidemic, and they also have the drawback of limited supply [6]. Thus far, evidence supporting the effectiveness of antivirals such as amantadine or neuraminidase inhibitors has not been well established [7]. Therefore, it is important to find other ways to reduce the prevalence of influenza infection.

The use of catechins and theanine, which are wellknown components of green tea, shows promise as an intervention for preventing influenza infection [8]. Experimental studies have revealed that green tea catechins can prevent influenza infection in vitro, but clinical evidence has so far been inconclusive [9]. Recently, in a small prospective cohort study, we reported that gargling with tea catechin extracts was effective in preventing influenza infection in elderly nursing home residents [10]. The consumption of tea extracts including catechins and theanine has also been reported to enhance systemic immunity and prevent the occurrence of upper respiratory tract infection and influenza symptoms in healthy adults [11]. Based on these findings, we designed a randomized, double-blind study to evaluate the clinical efficacy of green tea catechins and theanine in preventing influenza infection.

\section{Methods}

\section{Study Design}

A randomized, double-blind, 2-group parallel study was conducted to compare the effects of green tea catechins and theanine (catechin/theanine) capsules with those of placebo on the prevention of influenza for 5 months during the influenza season, from November 9, 2009 to April 8, 2010. We enrolled adult (over 20 years of age) healthcare workers who worked in 3 healthcare facilities for the elderly in Higashimurayama, Japan. Participants were excluded for the following criteria: tea allergy; history of influenza infection within 6 months before or 24 hours after entering the study; use of any medication or supplement affecting respiratory tract infections; immune disease; severe cardiac, respiratory, renal, or hepatic dysfunction; anemia requiring treatment; pregnancy or lactation.

The participants completed a self-administered questionnaire to assess baseline characteristics including age, sex, body mass index (BMI), smoking and alcohol consumption, and vaccination for the influenza virus. Their tea consumption habits before intervention were also determined, and any tea beverage such as black, green, oolong, or herbal tea intake was restricted to less than $250 \mathrm{~mL}$ per day over the entire course of the study.

Eligible participants were randomized by a computergenerated block randomization schema. Participants were provided with coded aluminum bags containing catechin/theanine capsules or placebo identical in appearance and taste in a double-blind manner. The participants were asked to take 6 capsules per day, containing a total of $378 \mathrm{mg}$ catechins (including $270 \mathrm{mg}$ (-)-epigallocatechin gallate) (THEA-FLAN 90S, ITO EN, Ltd., Tokyo, Japan) and $210 \mathrm{mg}$ theanine (Suntheanine ${ }^{\circledR}$, Taiyo Kagaku Co., Ltd., Mie, Japan) or placebo. The participants were asked to complete a questionnaire concerning the occurrence of influenza infection, preventive measures for maintaining hygiene, any adverse event, and their daily adherence to taking the capsules. The questionnaires were collected monthly, and careful safety monitoring was conducted throughout the study. Each participant was observed for the same time.

All participants gave written informed consent before entering the study. The study protocol was approved by the ethics committee at the University of Shizuoka and was conducted in accordance with the Declaration of Helsinki.

\section{Outcomes}

The primary outcome was the incidence of clinically defined influenza infection. The doctor diagnosed clinically defined influenza on the basis of fever (temperature, $\geq 37.8^{\circ} \mathrm{C}$ ) and any 2 of the following clinical symptoms: cough, sore throat, headache, or myalgia [12]. The secondary outcomes were (1) the incidence of laboratory-confirmed influenza infection with viral antigen measured by immunochromatographic assay (RapidTesta ${ }^{\circledR}$ FLUII, Sekisui Medical Co., Ltd., Tokyo, Japan) and (2) the time for which the patient was free from clinically defined influenza infection, i.e., the period between the start of the intervention and the first diagnosis of influenza infection. The antigen test was performed for all participants with suspected influenza infection, including clinically defined influenza. The antigen test, with a nasopharyngeal swab specimen, was approximately $85 \%$ sensitive and $100 \%$ specific for the influenza virus type A and B antigens $[10,13]$.

\section{Statistical Analyses}

In consideration of our previous study in the aged-group participants, we estimated that the primary outcome would occur in $1 \%$ of participants in the catechin/theanine group and $11 \%$ of those in the placebo group [10]. The sample size was calculated as 88 for each group at a power level of 
0.80 and a 2 -sided $\alpha$ level of 0.05 . With an estimated $10 \%$ dropout rate, we set the total sample size at 194.

All efficacy and safety analyses were performed according to the intention-to-treat principle. We used Fisher's exact test for categorical comparisons of the data. Differences in the mean values of continuous measurements were tested by Student's $t$ test or Wilcoxon rank sum test. Multiple logistic regression analysis was used to provide adjusted odds ratio (OR) estimates and 95\% confidence interval (CI) for association between catechin/ theanine capsules and the incidence of influenza infection. Cumulative incidence rates were determined by the Kaplan-Meier method. The Cox proportional hazards regression model was used to evaluate the association between catechin/theanine capsules and the time for which the patient was free from clinically defined influenza infection, adjusted for potential confounding variables after confirmation of the proportional hazard assumptions. The cases taken the influenza-free time to be censored were the occurrence of primary outcome and discontinuation. Among baseline characteristics, those with $P<0.20$ were considered potential confounding variables. They were defined on multiple logistic regression analysis and transferred to the Cox proportional hazard model. In these multivariable analyses, we also added age as a significant explanatory variable.

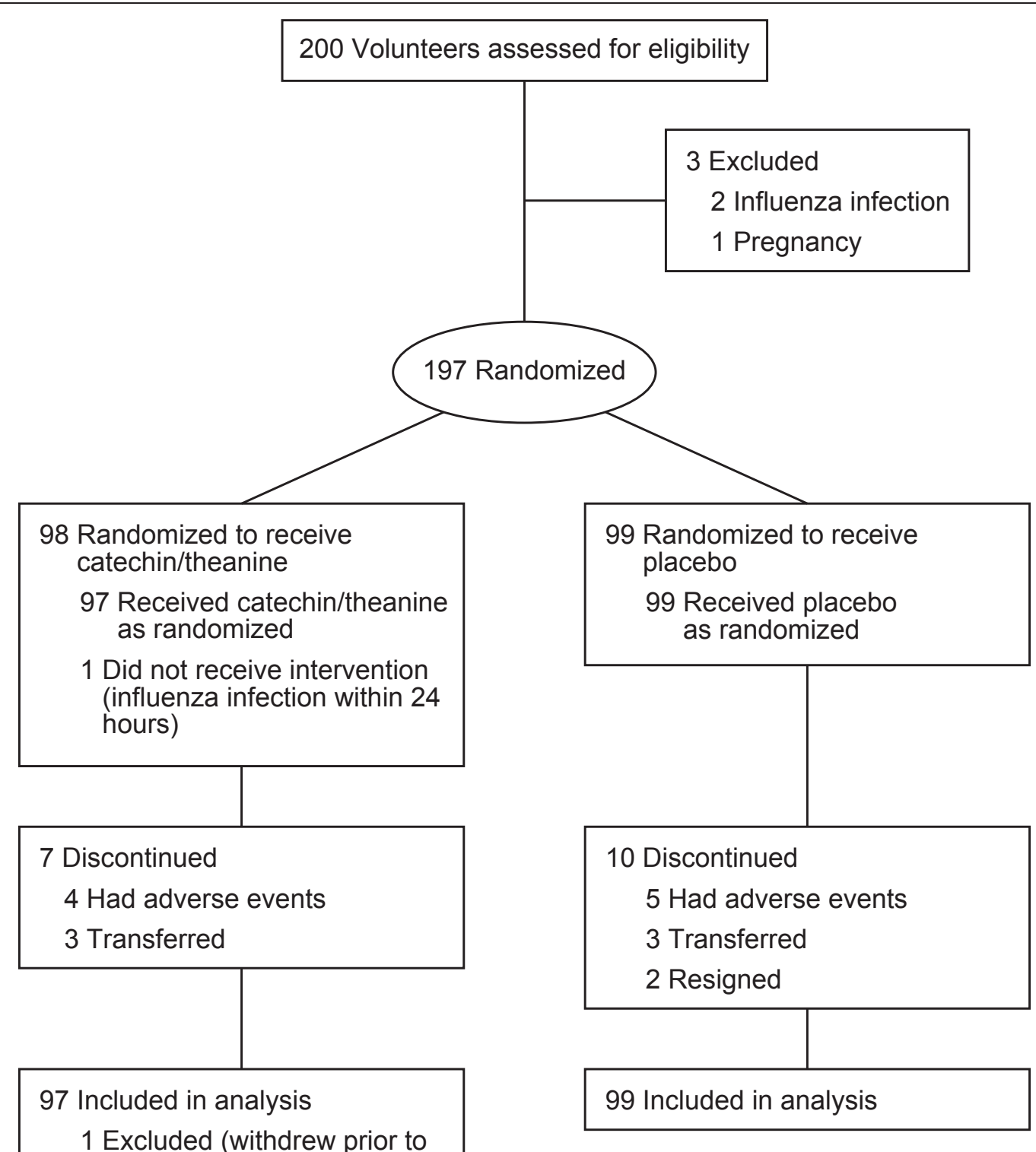

Figure 1 Flow diagram for trial of catechin/theanine vs. placebo. 
The threshold for statistical significance was set at $P<$ 0.05. Analyses were conducted using $\mathrm{R}$ version 2.11 .1 (R Foundation for Statistical Computing, Vienna, Austria).

\section{Results}

Of 200 participants recruited and assessed for eligibility, 3 were excluded according to the exclusion criteria (2, history of influenza infection within 6 months; 1 , pregnancy). The remaining 197 participants were enrolled and randomly assigned to an intervention; 98 were allocated to the catechin/theanine group and 99 to the placebo group (Figure 1). After assignment, 1 participant in the catechin/ theanine group was excluded according to the exclusion criteria (influenza infection within 24 hours after entering the study). Adherence to the test capsules was $93.2 \%$ in the catechin/theanine group and $91.9 \%$ in the placebo group.

The baseline characteristics of the participants are shown in Table 1. The mean age of participants was 42.7 years and uniformly distributed. Study groups were well balanced in terms of baseline characteristics except for BMI $(P=0.010)$. Therefore, BMI was considered as a potential confounding variable and was included in the variables of multivariate analyses. During the study, clinically defined influenza infection occurred in a total of 17 participants (8.7\%), 6 of whom (3.1\%) were laboratory confirmed with the type A antigen. No participants had more than one influenza infection during the observation time.

In the univariate comparison between the incidence of clinically defined influenza infections and baseline characteristics, age was the only significant variable and younger age was correlated with the high incidence of influenza infection $(P=0.027)$. Significant associations were not found among other variables such as sex, vaccination, preventive measures for maintaining hygiene (i.e., hand washing, facemask application, and gargling), smoking, and alcohol and tea consumption.

The incidence of clinically defined influenza infections was significantly lower in the catechin/theanine group (4 participants; $4.1 \%$ ) than in the placebo group (13 participants; $13.1 \%$ ) (adjusted OR, 0.25 ; 95\% CI, 0.07 to $0.76, P=0.022$ ). The incidence of laboratory-confirmed influenza infection was also lower in the catechin/theanine group (1 participant; $1.0 \%$ ) than in the placebo group (5 participants; $5.1 \%$ ), but this difference was not significant (adjusted OR, 0.17; 95\% CI, 0.01 to 1.10 ; $P=0.112$ ).

Kaplan-Meier curves were shown in Figure 2. The time for which the patient was free from clinically defined influenza infection, estimated with the Cox

Table 1 Baseline characteristics of study participants

\begin{tabular}{|c|c|c|c|}
\hline Characteristics & $\begin{array}{l}\text { Catechin/Theanine group } \\
\qquad(\mathrm{n}=97)\end{array}$ & $\begin{array}{l}\text { Placebo group } \\
(\mathrm{n}=99)\end{array}$ & $P$ Value \\
\hline Age, mean (SD) [range], y & $42.1(12.4)$ [21-65] & 43.2 (13.1) [22-69] & $0.63^{\mathrm{a}}$ \\
\hline \multicolumn{4}{|l|}{ Sex, No. (\%) } \\
\hline Male & $21(21.6)$ & $23(23.2)$ & \\
\hline Female & $76(78.4)$ & $76(76.8)$ & $0.86^{\mathrm{b}}$ \\
\hline BMI, mean (SD) [range] & $21.6(2.8)[15.9-30.9]$ & $22.7(3.2)[16.6-33.3]$ & $0.01^{c}$ \\
\hline Vaccination for influenza virus, No. (\%) & $91(93.8)$ & $91(91.9)$ & $>0.99^{b}$ \\
\hline Hand-washing ${ }^{d}$, No. (\%) & $72(74.2)$ & $72(72.7)$ & $0.87^{b}$ \\
\hline Facemasks $^{\mathrm{d}}$, No. (\%) & $15(15.5)$ & $20(20.2)$ & $0.46^{\mathrm{b}}$ \\
\hline Gargling ${ }^{\mathrm{d}}$, No. (\%) & $71(73.2)$ & $68(68.7)$ & $0.53^{\mathrm{b}}$ \\
\hline \multicolumn{4}{|l|}{ Smoking, No. (\%) } \\
\hline Yes & $29(29.9)$ & $29(29.3)$ & \\
\hline Past & $15(15.5)$ & $15(15.2)$ & $>0.99^{b}$ \\
\hline No & $53(54.6)$ & 55 (55.6) & \\
\hline \multicolumn{4}{|l|}{ Alcohol consumption, No. (\%) } \\
\hline Yes & $49(50.5)$ & $56(56.6)$ & \\
\hline Past & $3(3.1)$ & $4(4.0)$ & $0.66^{\mathrm{b}}$ \\
\hline No & $45(46.4)$ & $39(39.4)$ & \\
\hline Tea consumption, mean (SD), mL/day & $494.3(415.6)$ & $481.3(374.3)$ & $0.95^{\mathrm{a}}$ \\
\hline $\begin{array}{l}\text { Abbreviation: BMI, body mass index. } \\
{ }^{\mathrm{a}} P \text { value based on Wilcoxon rank sum test. } \\
{ }^{\mathrm{b}} P \text { value based on Fisher's exact test. } \\
{ }^{{ }^{C} P} \text { value based on Student's } t \text { test. }\end{array}$ & & & \\
\hline
\end{tabular}




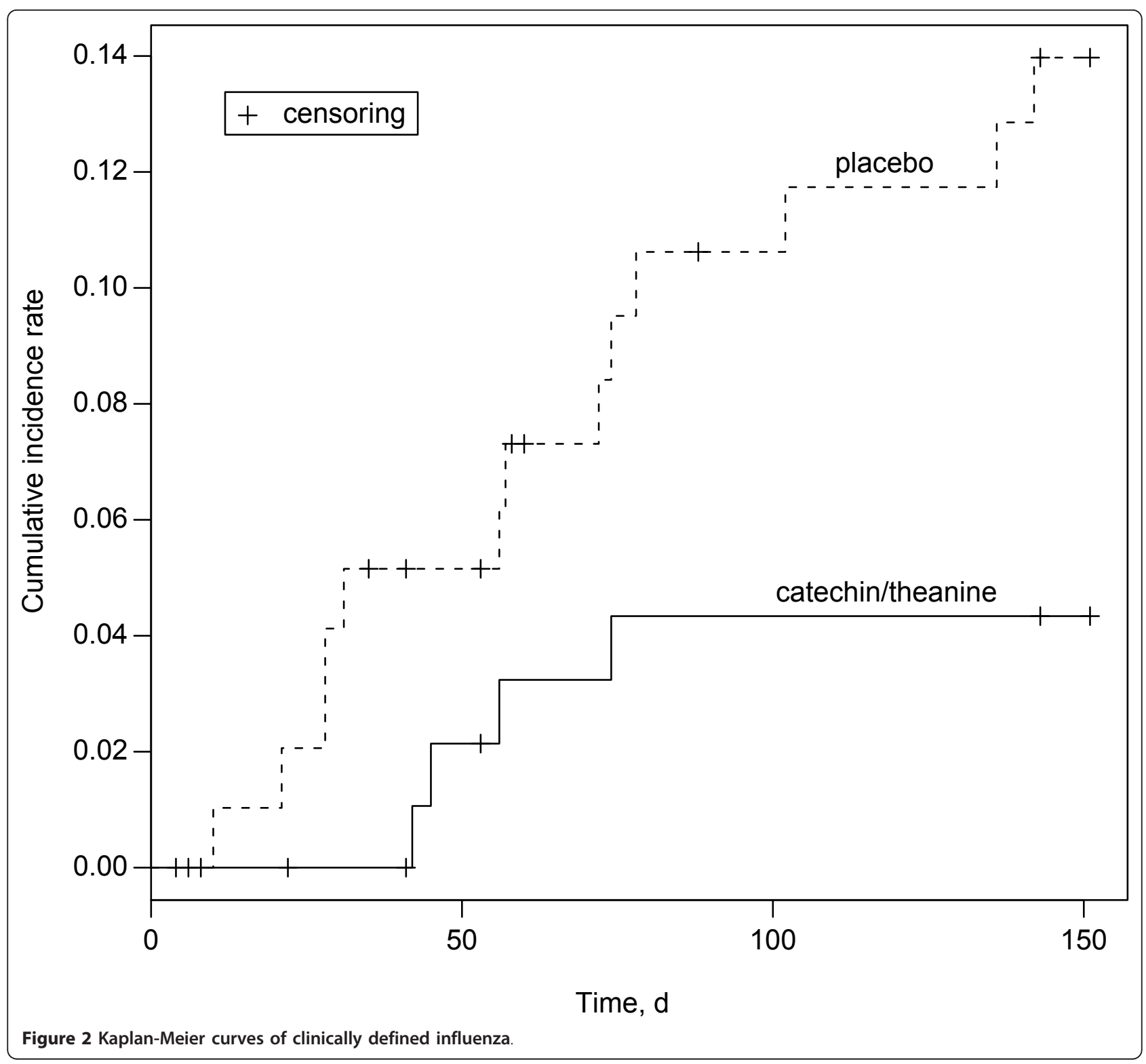

Table 2 Results of multiple logistic regression analysis and Cox proportional hazards regression model

\begin{tabular}{|c|c|c|c|c|c|c|}
\hline \multirow[b]{2}{*}{ Variables } & \multicolumn{2}{|c|}{ Laboratory confirmed influenza $^{a}$} & \multicolumn{4}{|c|}{ Clinically defined influenza ${ }^{b}$} \\
\hline & OR $(95 \% \mathrm{Cl})^{\mathrm{c}}$ & $P$ Value $^{c}$ & OR $(95 \% \mathrm{Cl})^{\mathrm{c}}$ & $P$ Value $^{c}$ & $\operatorname{HR}(95 \% \mathrm{Cl})^{d}$ & $P$ Value ${ }^{d}$ \\
\hline \multicolumn{7}{|l|}{ Allocation } \\
\hline Catechin/Theanine & $0.17(0.01$ to 1.10$)$ & 0.11 & 0.25 (0.07 to 0.76$)$ & 0.02 & 0.27 (0.09 to 0.84$)$ & 0.02 \\
\hline Placebo & 1 [Reference] & & 1 [Reference] & & 1 [Reference] & \\
\hline Age & 0.98 (0.91 to 1.04$)$ & 0.48 & 0.95 (0.91 to 0.99) & 0.03 & 0.96 (0.92 to 1.00$)$ & 0.04 \\
\hline $\mathrm{BMI}$ & 0.89 (0.63 to 1.16$)$ & 0.43 & $0.95(0.79$ to 1.11$)$ & 0.53 & 0.97 (0.83 to 1.13$)$ & 0.66 \\
\hline
\end{tabular}

Abbreviations: $\mathrm{OR}$, odds ratio; $\mathrm{Cl}$, confidence interval; $\mathrm{HR}$, hazard ratio; $\mathrm{BMI}$, body mass index.

a Laboratory-confirmed influenza infection with viral antigen detected by immunochromatographic assay.

${ }^{\mathrm{b}} \mathrm{Clinically} \mathrm{defined} \mathrm{influenza} \mathrm{was} \mathrm{diagnosed} \mathrm{as} \mathrm{fever} \mathrm{(temperature,} \geq 37.8^{\circ} \mathrm{C}$ ) and any 2 of the following clinical symptoms: cough, sore throat, headache, or myalgia.

${ }^{\mathrm{C}} \mathrm{OR}$ and $P$ values were estimated using multiple logistic regression.

${ }^{\mathrm{d}} \mathrm{HR}$ and $P$ values were estimated using the Cox proportional hazards regression model. 
proportional hazards regression model, was significantly different between the two groups (adjusted hazard ratio, 0.27 ; $95 \% \mathrm{CI}, 0.09$ to $0.84 ; P=0.023$ ) (Table 2). No serious adverse events were observed during the study. Digestive symptoms such as bloating and constipation occurred in both groups and were reported by $9.2 \%$ of all participants. These symptoms were relativity mild and did not significantly differ between the two groups.

\section{Discussion and Conclusion}

This randomized, double-blind, placebo-controlled trial was conducted to determine whether taking green tea catechins and theanine could clinically prevent influenza infection. We found that consuming catechin/theanine for 5 months had a statistically significant preventive effect on clinically defined influenza infection and was well tolerated. To our knowledge, this is the first randomized clinical trial to evaluate the efficacy of green tea catechins and theanine in the prevention of influenza infection.

Experimental studies have shown some mechanisms of the action of green tea catechins and theanine on the prevention of influenza infection. These studies have shown that green tea catechins bind to the hemagglutinin molecule of influenza virus, thereby inhibiting the virus adsorption to the host cells and blocking virus assembly or maturation cleavage [14-16]. Theanine has a possibility to enhance the systemic immunity $(\gamma \delta \mathrm{T}$ cell function) for influenza infection [11]. Our results seem to provide clinical evidence to confirm these biological activities.

Contrary to worldwide prevalence in the 2009 novel influenza virus A (H1N1) pandemic, laboratory-confirmed influenza infection occurred in only $3.1 \%$ of the study participants. Probably in part due to this small sample size, the incidence of laboratory-confirmed influenza was not significantly different in the catechin/theanine group, in spite of showing a decreased tendency compared to placebo. Moreover, the participants were healthcare workers with a high rate $(92.9 \%)$ of vaccination; therefore the effectiveness of catechin/theanine might be underestimated. Additional large-scale randomized trials are needed to confirm the effectiveness of catechin/theanine as prophylaxis for laboratory-confirmed influenza infection.

\section{Abbreviations}

OR: odds ratio; Cl: confidence interval; HR: hazard ratio.

\section{Acknowledgements}

We gratefully acknowledge the participants who participated in the study, the clinical research coordinators, and other medical staff at the University of Shizuoka (Mr. Keisuke Shiozawa, Mr. Kiichiro Toyoizumi, Ms. Mijong Park, Mr.
Kazuhiro Kosuge), White Cross Nursing Home (Ms. Sadako Fujii, Ms. Yumiko Yahata), and Tokyo White Cross Hospital (Ms. Kyoko Onogi, Ms. Mitsuko Ogawa) for their dedicated cooperation.

\section{Author details}

${ }^{1}$ Department of Drug Evaluation \& Informatics, Graduate School of Pharmaceutical Sciences, University of Shizuoka, 52-1 Yada, Suruga-ku, Shizuoka 422-8526, Japan. 'White Cross Nursing Home, Higashimurayama, Japan. ${ }^{3}$ Central Research Institute, ITO EN, Ltd., Shizuoka, Japan.

\section{Authors' contributions}

KM had complete access to all the data in the study, and he is responsible for the integrity of the data and the accuracy of the data analysis. HY designed the study protocol and participated in its coordination. NT participated in the study coordination. HN and YMS participated in the design of the study and provided the experimental supplements. All authors read and approved the final manuscript.

\section{Competing interests}

This work was supported by a grant from the Japanese Ministry of Health, Labor, and Welfare, and a grant from ITO EN Ltd. ITO EN Ltd played a role in providing the experimental supplements.

Received: 13 October 2010 Accepted: 21 February 2011

Published: 21 February 2011

\section{References}

1. Thompson WW, Shay DK, Weintraub E, et al: Influenza-associated hospitalizations in the United States. JAMA 2004, 292:1333-1340.

2. Bell DM, World Health Organization Writing Group: Non-pharmaceutical interventions for pandemic influenza, national and community measures. Emerg Infect Dis 2006, 12:88-94.

3. Aiello AE, Murray GF, Perez $V$, et al: Mask use, hand hygiene, and seasonal influenza-like illness among young adults: a randomized intervention trial. J Infect Dis 2010, 201:491-498.

4. Aledort JE, Lurie N, Wasserman J, Bozzette SA: Non-pharmaceutical public health interventions for pandemic influenza: an evaluation of the evidence base. BMC Public Health 2007, 7:208.

5. Cowling BJ, Chan $\mathrm{KH}$, Fang VJ, et al: Facemasks and hand hygiene to prevent influenza transmission in households: a cluster randomized trial. Ann Intern Med 2009, 151:437-446.

6. Collin N, de Radiguès X, World Health Organization H1N1 Vaccine Task Force: Vaccine production capacity for seasonal and pandemic (H1N1) 2009 influenza. Vaccine 2009, 27:5184-5186.

7. Jefferson T, Jones M, Doshi P, Del Mar C: Neuraminidase inhibitors for preventing and treating influenza in healthy adults: systematic review and meta-analysis. BMJ 2009, 339:b5106.

8. Balentine DA, Wiseman SA, Bouwens LC: The chemistry of tea flavonoids. Crit Rev Food Sci Nutr 1997, 37:693-704.

9. Song JM, Lee KH, Seong BL: Antiviral effect of catechins in green tea on influenza virus. Antiviral Res 2005, 68:66-74.

10. Yamada H, Takuma N, Daimon T, Hara Y: Gargling with tea catechin extracts for the prevention of influenza infection in elderly nursing home residents: a prospective clinical study. J Altern Complement Med 2006, 12:669-672.

11. Rowe CA, Nantz MP, Bukowski JF, Percival SS: Specific formulation of Camellia sinensis prevents cold and flu symptoms and enhances gamma,delta T cell function: a randomized, double-blind, placebocontrolled study. J Am Coll Nutr 2007, 26:445-452.

12. Boivin G, Hardy I, Tellier G, Maziade J: Predicting influenza infections during epidemics with use of a clinical case definition. Clin Infect Dis 2000, 31:1166-1169.

13. Maezawa T, Yamamura $Y$, Suganuma T: Evaluation of a rapid detection kit for influenza $A$ and $B$ viruses using immunochromatography "RapidTesta® FLU II". Igaku Yakugaku 2004, 52:481-486.

14. Mukhtar $\mathrm{H}$, Ahmad N: Tea polyphenols: prevention of cancer and optimizing health. Am J Clin Nutr 2000, 71:1698S-1702S. 
15. Mantani $\mathrm{N}$, Imanishi $\mathrm{N}$, Kawamata $\mathrm{H}$, Terasawa $\mathrm{K}$, Ochiai $\mathrm{H}$ : Inhibitory effect of (+)-catechin on the growth of influenza A/PR/8 virus in MDCK cells. Planta Med 2001, 67:240-243.

16. Imanishi $\mathrm{N}$, Tuji $\mathrm{Y}$, Katada $\mathrm{Y}$, Maruhashi M, Konosu S, Mantani N, Terasawa $\mathrm{K}$, Ochiai H: Additional inhibitory effect of tea extract on the growth of influenza A and B viruses in MDCK cells. Microbiol Immunol 2002, 46:491-494.

\section{Pre-publication history}

The pre-publication history for this paper can be accessed here: http://www.biomedcentral.com/1472-6882/11/15/prepub

doi:10.1186/1472-6882-11-15

Cite this article as: Matsumoto et al:: Effects of Green Tea Catechins and Theanine on Preventing Influenza Infection among Healthcare Workers: A Randomized Controlled Trial. BMC Complementary and Alternative Medicine 2011 11:15.

Submit your next manuscript to BioMed Central and take full advantage of:

- Convenient online submission

- Thorough peer review

- No space constraints or color figure charges

- Immediate publication on acceptance

- Inclusion in PubMed, CAS, Scopus and Google Scholar

- Research which is freely available for redistribution

Submit your manuscript at www.biomedcentral.com/submit
() Biomed Central 